\section{Kongressankündigungen Congress Announcements}

\section{4. Österreichischer HNO-Kongress 2010}

Termin: 15.-18. September 2010

Ort: Salzburg, Universität Mozarteum

Veranstalter: Österreichische Gesellschaft für Hals-, Nasen- und Ohrenheilkunde, Kopf- und Halschirurgie; unter den Auspizien der European Academy of Oto-Rhino-Laryngology, Head and Neck Surgery

Kongresspräsidenten: Prim. Univ.-Prof. Dr. Gerd Rasp, Universitätsklinik für HNO-Krankheiten, Paracelsus Medizinische Privatuniversität, Müllner Hauptstraße 48, 5020 Salzburg, Tel.: ++43-662-4482-4000; Fax: ++43-662-4482-4033, E-mail: g.rasp @salk.at

Hauptthema: Form und Rekonstruktion - Workshops: Allergie Schwindel - Audiologie - Traumatologie - Simulation

Gesellschaftssekretariat: Mondial Congress \& Events, Mag. Andrea Balcar, Operngasse 20b, 1040 Wien, Tel.: ++43-158804-800, Fax: ++43-1-58804-185, E-mail: sekretariat@hno.at, Homepage: www.hno.at

\section{Jahrestagung der Österreichischen Gesellschaft für Innere Medizin}

Termin: 29. September-2. Oktober 2010

Tagungsort: Congress Salzburg

Generalthema: Der internistische Akutpatient - zwischen Klinik und Praxis

Tagungspräsident: Univ.-Prof. Dr. Kurz Lenz, Interne Abteilung, Konventhospital der Barmherzigen Brüder Linz, Seilerstätte 2, 4020 Linz, Tel.: $++43-732-78$ 97-24301, Fax: $++43-732-78$ 9724398, E-mail: kurz.lenz@bblinz.at

Tagungssekretär: Univ.-Prof. Dr. Christian Madl, 4. Medizinische Abteilung, Krankenanstalt Rudolfstiftung, Juchgasse 25, 1030 Wien, Tel.: $++43-1-711$ 65-2407, Fax: $++43-1-711$ 65-2409, E-mail: christian.madl@wienkav.at, Homepage: www.oegim.at

\section{Jahrestagung 2010 der Österreichischen Gesellschaft für Pneumologie \\ Termin: 7.-9. Oktober 2010}

Tagungsort: Graz, Stadthalle

Veranstalter: Österreichische Gesellschaft für Pneumologie Hauptthemen: Endotheliale Funktion - Cor Pulmonale Kongressleitung und ÖGP-Präsident: Univ.-Prof. Dr. Horst Olschewski, Leiter der Klinische Abteilung für Lungenkrankheiten, Universitätsklinik für Innere Medizin, Medizinische Universität Graz, Auenbruggerplatz 20, 8036 Graz, Tel.: ++43316-385-2183, Fax: ++43-316-385-3578, E-mail: horst. olschewski@medunigraz.at

Kongresssekretäre: OA Dr. Gabor Kovacs und OA Dr. Stefan Scheidl, Klinische Abteilung für Lungenkrankheiten, Universitätsklinik für Innere Medizin, Medizinische Universität Graz Auenbruggerplatz 20, 8036 Graz, Tel.: ++43-316-385-80748, $++43-316-385-80288$, Fax: $++43-316-316-385-17034$, E-mail: gabor.kovacs@klinikum-graz.at, stefan.scheidl@klinikum-graz.at, Homepage: www.ogp.at

\section{Jahrestagung der Österreichischen Adipositas Gesellschaft} Termin: 29.-30. Oktober 2010

Tagungsort: Schloss Seggau

Veranstalter: Österreichische Adipositas Gesellschaft Hauptthemen: VLCD-s im Focus - maßgeschneiderte Bewegungstherapie - Adipositas und Arbeitsplatz - Adipositas im Kinder- und Jugendalter - Homo metabolicus - gewichtsneutrale Diabetestherapie - Gender hat Gewicht - Adipositasparadoxon gesunde Lebensmittel im Focus - nationaler Aktionsplan Adipositas? - integrierte Versorgung und bariatrische Chirurgie Kongresspräsidentin und ÖAG-Präsidentin: Univ.-Prof. Dr. Anita Rieder, Institut für Sozialmedizin, Zentrum für Public Health, Medizinische Universität Wien, Rooseveltplatz 3, 1090 Wien, Tel.: $++43-1-4277-646$ 80, Fax: ++43-1-4277-646 81, E-mail: anita. rieder@meduniwien.ac.at

Tagungsbüro: Österreichische Adipositasgesellschaft, Sekretariat: Frau Simone Posch, Währinger Straße 76/13, 1090 Wien, Tel.: $++43-650-77033$ 78, Fax: ++43-1-264 52 29, E-mail: office@ adipositas-austria.org, Homepage: www.adipositas-austria.org

\section{Jahrestagung der Österreichischen Diabetes Gesellschaft Termin: 18.-20. November 2010}

Tagungsort: Salzburg Congress Veranstalter: Österreichische Diabetes Gesellschaft

Hauptthemen: „Translation of Science - von der Forschung in die Praxis": Diabetes und Lebensstil - Neues zu diabetischen Folgeerkrankungen: Auge, Nerven, Niere - Diabetes und Krebserkrankungen - DMP-Update - Neue Medikamente kritisch betrachtet - Diabeteslabor im Wandel - Neues zu Diabetes und Schwangerschaft - Psychiatrische und psychosomatische Begleiterkrankungen bei Kindern und Jugendlichen mit Typ 1 Diabetes Wissenschaftliche Leitung und ÖDG-Präsident: Univ.-Doz. Dr. Raimund Weitgasser, Leiter Schwerpunkt Diabetologie, Universitätsklinik für Innere Medizin I, Paracelsus Medizinische Privatuniversität, Müllner Hauptstraße 48, 5020 Salzburg, Tel.: $++43-662-4482-3423, \quad$ Fax: $\quad++43-662-4482-881, \quad$ E-mail: r.weitgasser@salk.at

1. Sekretär der ÖDG: Univ.-Doz. Dr. Bernhard Paulweber, Universitätsklinik für Innere Medizin I, Paracelsus Medizinische Privatuniversität, Müllner Hauptstraße 48, 5020 Salzburg, E-mail: b.paulweber@salk.at, Homepage: www.oedg.org

\section{Jahrestagung der Österreichischen Gesellschaft für Dermatologie und Venerologie Termin: 19.-21. November 2010}

Tagungsort: Wien, Aula der Wissenschaften, Wollzeile 27a, 1010 Wien

Veranstalter: Österreichische Gesellschaft für Dermatologie und Venerologie

Wissenschaftliche Leitung und ÖGDV-Präsident: Prim. Univ.Prof. Dr. Beatrix Volc-Platzer, Dermatologische Abteilung, Sozialmedizinisches Zentrum Ost - Donauspital, Langobardenstraße 122, 1220 Wien, Tel.: ++43-1-288 02-4100, Fax: ++43-1-288 024180, E-mail: beatrix.volc-platzer@wienkav.at 